\title{
Neoadjuvante Chemotherapie beeinflusst das Tumorprofil bei Brustkrebs?
}

Welchen Einfluss hat eine neoadjuvante Chemotherapie auf die Tumorcharakteristika eines Mammakarzinoms. Bisherige Daten geben widerprüchliche Hinweise. Nun liegen Ergebnisse einer britischen Arbeitsgruppe vor.

A ufgrund der Tumorcharakteristika unterzogen sich 246 Brustkrebspatientinnen mit primär operablem Tumor einer neoadjuvanten Chemotherapie (NACT). Renu Gahlaut und Kollegen verglichen die Charakteristika des Tumorbiopsie vor NACT mit denen der posttherapeutischen Tumorresiduen. Dabei berücksichtigten sie neben Tumortyp und -grading auch den Hormonrezeptor- und HER2-Status. Die Biopsien vor NACT ergaben folgendes Bild: $86,5 \%$ Karzinome ohne speziellen Typ 48,6\% Grad-3-Tumoren, 64,9\% ER-po-
Tab. 1: Einfluss der NACT auf den Rezeptorstatus

ER (prä-NACT) ER (post-NACT)

\begin{tabular}{|c|c|c|c|c|c|c|c|c|c|}
\hline & 0 & 2 & 3 & 4 & 5 & 6 & 7 & 8 & gesamt \\
\hline 0 & 36 & $3^{b}$ & 3 & $2^{\mathrm{a}}$ & $1^{\mathrm{a}}$ & 0 & $1^{\mathrm{a}}$ & $2^{\mathrm{a}}$ & 48 \\
\hline 2 & 3 & 0 & 0 & $1^{a}$ & 0 & 0 & 0 & 0 & 4 \\
\hline 3 & 2 & 0 & 1 & 0 & 0 & 0 & $1^{b}$ & 0 & 4 \\
\hline 4 & $2^{\mathrm{a}}$ & 0 & 0 & 0 & 0 & 0 & 0 & $1^{b}$ & 3 \\
\hline 5 & 0 & 0 & $1^{b}$ & 0 & 2 & 0 & 0 & $2^{b}$ & 5 \\
\hline 6 & 0 & 0 & 0 & 0 & 4 & 0 & 2 & $2^{b}$ & 8 \\
\hline 7 & $1^{\mathrm{a}}$ & 0 & 0 & $3^{b}$ & $1^{b}$ & 3 & 3 & 9 & 20 \\
\hline 8 & $2^{\mathrm{a}}$ & $1^{\mathrm{a}}$ & 0 & $1^{b}$ & $2^{b}$ & $1^{b}$ & 7 & 27 & 41 \\
\hline gesamt & 46 & 4 & 5 & 7 & 10 & 4 & 14 & 43 & 133 \\
\hline
\end{tabular}

$E R=$ Östrogenrezeptor; $N A C T=$ neoadjuvante Chemotherapie; ${ }^{a}$ Wechsel des ER-Status nach NACT: von positiv auf negative oder umgekehrt; ${ }^{b}$ Deutliche Änderung des Expressionslevels des Rezeptors ohne Änderung des finalen ER-Status. sitive Tumoren und 19,9\% HER2-positive Tumoren. 53 Frauen erreichten mit NACT eine komplette pathologische Remission und wurden von der weiteren Analyse ausgeschlossen. Nach der NACT musste in 29 von 178 Fällen die histologische Typisierung korrigiert werden ( $\mathrm{p}<0,001)$, wobei der Anteil lobulärer Adenokarzinome und metaplastischer Karzinome gestiegen war. Bei $28,8 \%$ war das Tumorgewebe nach Chemotherapie besser differenziert. Eine Änderung im ER-, PR- bzw. HER2-Status war bei $12 \%$, $14,5 \%$ und 7,1\% der Tumoren nachweisbar, v.a. ein Wechsel von negativem zu positivem ER- und von positivem zu negativem HER2-Status (Tab. 1).

Fazit: Eine NACT verursacht signifikante Veränderungen in Morphologie, Grading und Rezeptorstatus des Tumorgewebes, sodass eine erneute $\mathrm{Tu}$ morcharakterisierung sinnvoll sein könnte, weil dies durchaus therapeutische Konsequenzen haben könnte.

Dagmar Kraus

Gahlaut R et al. Effect of neoadjuvant chemotherapy on breast cancer phenotype, ER/PR and HER2 expression e Implications for the practising oncologist. Eur J Cancer. 2016;60:40-8.

\section{Endokrine Therapie: besseres Nebenwirkungsmanagement wichtig}

In der Mind-Body-Studie wurden Lebensqualität und Symptomlast von Brustkrebspatientinnen mit und ohne endokrine Therapie (ET) nach der Primärtherapie verglichen.

Vosentar on 186 Patientinnen mit abgeschlossener Primärtherapie bekamen 60 keine ET, 66 Tamoxifen (TAM) und 60 Aromataseinhibitoren (AI). Zu Beginn, nach 6 und 12 Monaten wurden die Frauen nach ihrem Befinden befragt. Genutzt wurden die Short Form 36 (SF36), die BCPT(Breast Cancer Prevention Trial)Symptomskala für ET-typische Symptome sowie spezifische Instrumente für Depression, Fatigue und Schlafstörungen.
Der physische Funktionswert des SF-36 verbesserte sich im Verlauf der Studie in allen Gruppen leicht, in der Gruppe mit AI-Therapie nach 12 Monaten aber weniger als in der Gruppe ohne ET. Der mentale Funktionswert des SF-36 war zu Beginn im normalen Bereich und änderte sich über 12 Monate kaum. Es ergaben keine Unterschiede zwischen den Gruppen. Die Symptomschwere nach der BCPT-Skala war in der Gruppe ohne ET stabil oder besserte sich im Verlauf, während die einzelnen Symptome in den ETGruppen teilweise noch zunahmen. Die AI-Gruppe entwickelte nach 6 und $12 \mathrm{Mo-}$ naten besonders ausgeprägte muskuloskeletale Symptome ( $\mathrm{p}=0,02$ vs. keine ET), außerdem hatte sie eine höhere Belastung durch Hitzewallungen ( $p=0,02$ vs. keine ET) und kognitive Probleme ( $\mathrm{p}=0,006$ vs. keine ET). Auch die TAM-Gruppe litt stark unter Hitzewallungen $(\mathrm{p}=0,002)$, kognitiven Problemen $(\mathrm{p}=0,016)$ und Harnblasenbeschwerden $(\mathrm{p}=0,02)$.

Fazit: Der erhöhten Symptomlast unter einer ET sollte mehr Aufmerksamkeit geschenkt werden - in der Aufklärung, im Nebenwirkungsmanagement und in weiteren Therapiestudien. Friederike Klein

Ganz PA et al. Impact of Adjuvant Endocrine Therapy on Quality of Life and Symptoms: Observational Data Over 12 Months From the MindBody Study. J Clin Oncol. 2016;34(8):816-24. 